médecine/sciences $1994 ; 10: 742-3$

\title{
L'ADN ribosomique de Entamoeba histolytica code sur son brin complémentaire pour des facteurs de virulence, les hémolysines
}

La dysenterie amibienne résulte de la destruction de l'épithélium colique par le pathogène humain Entamoeba histolytica. Pour cela, le parasite séjourne tout d'abord dans le gros intestin où il colonise la surface muqueuse. La pénétration du mur colique peut conduire à la dissémination de l'amibe à d'autres organes, essentiellement le foie, avec formation d'abcès. Les facteurs de virulence impliqués dans la lyse des cellules hôtes comprennent, d'une part, l'amoebaporine, peptide de 77 acides aminés formant des pores; ce peptide possède un motif de six résidus cystéine, commun aux saposines et à la protéine $\mathrm{B}$ associée au surfactant, dont le rôle serait de stabiliser une structure tertiaire du peptide favorable à son action [1] ; et, d'autre part, des protéines hémolytiques. $\mathrm{Si}$ les mécanismes d'action de l'amoebaporine commencent à être connus, les bases moléculaires de la cytolyse par les protéines hémolytiques ne sont pas bien définies.

Une équipe suédoise vient d'isoler des gènes d'hémolysines chez $E$. histolytica [2]. Ils ont pour cela criblé une banque phagique d'expression chez Escherichia coli. La banque a été préparée à partir d'ADN génomique d'une souche de E. histolytica pathogène. La lyse des bactéries hôtes par les phages libère les pro- être détectées. Les clones comprenant des gènes codant pour des hémolysines ont été identifiés par visualisation directe d'une zone d'hémolyse sur gélose au sang. Avec cette approche, les gènes peuvent être isolés et les facteurs de virulence caractérisés chez $E$. coli, sans purification des protéines à partir du parasite. Quatre gènes candidats codant pour des hémolysines ont ainsi été identifiés. Les extraits protéiques des clones hémolytiques sont toxiques pour les cellules intestinales humaines Caco-2, premières cibles cellulaires de l'invasion amibienne dans le gros intestin. Ces gènes sont répartis sur un fragment de 2,6 kb et dépourvus d'introns. HLY4 est composé de 102 codons, HLYl de 37, et deux cadres ouverts de lecture ont été identifiés dans l'insert pHLY5m, l'un de 58 codons et l'autre de 30 codons. Aucune similitude avec une quelconque protéine connue à ce jour n'a été mise en évidence. En revanche, l'analyse de la séquence de ce locus d'hémolysines a révélé qu'il était identique à l'ARN ribosomique (ARNr) d'E. histolytica. HLY4 est localisé dans la région intergénique entre les gènes d'ARNr de la petite sous-unité et de l'ARNr 5,8S, alors que les autres gènes, HLYl et HLY5m, sont situés au niveau du gène d'ARNr de la grande sousunité. Chose remarquable, ces gènes
HLY sont transcrits dans la direction opposée à celle des ARNr. Cela est à rapprocher de ce que l'on observe au niveau du génome mitochondrial de Chlamydomonas où les gènes codant pour l'ARNr et pour les protéines de la chaîne respiratoire sont cotranscrits à partir des deux brins de l'ADNr. L'ADNr de E. histolytica est organisé sous forme d'un motif (ARNr de la petite sousunité - ARNr 5,8S - ARNr de la grande sous-unité) répété à l'envers. Cette répétition d'ADNr est portée par un plasmide de $25 \mathrm{~kb}$ dont il existe à peu près 200 copies par cellule.

Cinq cents millions de personnes sont, de par le monde, infectées par E. histolytica mais seulement $10 \%$ d'entre elles vont développer des symptômes de maladie invasive. Les souches pathogènes d'E. histolytica, isolées de cas symptomatiques sont génétiquement distinctes des souches provenant des porteurs asymptomatiques. En effet, le profil de migration des enzymes métaboliques (hexokinase et phosphoglucomutase) permet de définir un zymodème* pathogène et un zymodème non pathogène. En outre, des différences au niveau des séquences d'ADN génomique ont été retrou-

\footnotetext{
* Zymodène: population d'amibes présentant un mêrne profil électrophorétique d'enzymes cytoplasmiques.
} 
vées entre les souches invasives et non invasives [3]. Une particularité du plasmide portant l'ADNr est qu'il existe, entre les deux motifs d'ADNr, une séquence répétée de 140 paires de bases. Cette séquence est différente $(43,6 \%$ de similarité) selon que les souches sont pathogènes ou non $[4,5]$. Cela suggère que cette séquence répétée pourrait être impliquée dans l'expression des hémolysines. De plus, le profil de restriction (RFLP) de l'ADNr de la petite sous-unité ribosomique est différent selon que les souches entraînent des manifestations cliniques ou non [6]. Les auteurs ont ainsi montré que HLY4 n'était présent que chez les souches pathogènes [2]. Ce travail établit un lien direct entre l'ADNr et des gènes de virulence, expliquant ainsi comment des différences dans les séquences d'ARNr peuvent influer sur l'évolution de l'infection amibienne.

E.D.

1. Leippe M, Tannich E, Nickel R, Van der Goot G, Pattus F, Horstmann RD, MullerEberhard HJ. Primary and secondary structure of the pore-forming peptide of pathogenic Entamoeba histolytica. EMBO J $1992 ; 11$ : 3501-6.

2. Jansson A, Gillin F, Kagardt U, Hagblom P. Coding of hemolysins within the ribosomal RNA repeat on a plasmid in Entamoeba histolytica. Science 1994; 263 : 1440-3.

3. Tannich E, Horstmann RD, Knobloch J, Arnold HH. Genomic DNA differences between pathogenic and nonpathogenic Entamoeba histolytica. Proc Natl Acad Sci USA 1989 ; 86: $5118-22$.

4. Garfinkel LI, Giladi M, Huber M, Gitler C, Mirelman D, Revel M, Rozenblatt S. DNA probes specific for Entamoeba histolytica possessing pathogenic and nonpathogenic zymodemes. Infect Immun 1989; 57 : 926-31.

5. Bracha R, Diamond LS, Ackers JP, Burchard GD, Mirelman D. Differentiation of clinical isolates of Entamoeba histolytica by using specific DNA probes. Infect Immun 1991; 28 : 680-4.

6. Que X, Reed SL. Nucleotide sequence of a small subunit ribosomal RNA (I6S-like rRNA) gene from Entamoeba histolytica differentiation of pathogenic from nonpathogenic isolates. Nucleic Acids Res 1991 ; 19: 5438.

$\begin{array}{llll}m / s & n^{\circ} & 6-7 & \text { vol. } 10 \text {, juin-juillet } 94\end{array}$

\section{BRÈVES}

Les effets différentiels des apolipoprotéines E3 et E4 sur la croissance des neurites in vitro. L'isoforme apoE4 de l'apolipoprotéine $\mathrm{E}$ constitue un important facteur de risque d'un développement précoce d'une maladie d'Alzheimer ( $m / s \quad n^{\circ} 10$, vol.9, p. 1142). L'apoE est l'une des apolipoprotéines majeures dans le cerveau et dans le liquide céphalorachidien. Elle est associée aux plaques séniles et aux faisceaux neurofibrillaires observés dans des prélèvements post-mortem de malades atteints d'Alzheimer. Nathan et al., de San Francisco (CA, USA), viennent maintenant de montrer que l'apoE3 et l'apoE4 avaient des effets très différents sur la croissance neuritique au niveau de neurones en culture [1]. Le système utilisé est celui des cellules de ganglions de la racine dorsale de lapin. L'addition de particules VLDL $\beta$ de lapin ( $\beta$-migrating very low density lipoproteins) stimule très fortement une croissance neuritique ramifiée. L'addition à VLDL $\beta$ de l'apoE3 humaine purifiée diminue la ramification, mais augmente de manière importante la taille des neurites. En revanche, l'addition d'apoE4 humaine à faible concentration inhibe à la fois la croissance et la ramification des neurites. Les VLDL $\beta$ de lapin interagissent avec le récepteur des LDL (low density lipoproteins) présents à la surface des neurones. L'effet des isoformes d'apolipoprotéines E passe par une interaction avec les VLDL $\beta$ car il ne s'exerce pas en leur absence et est inhibé par des anticorps empêchant la fixation de l'apo E aux VLDL. Ces résultats complètent les différences déjà observées entre apoE3 et apoE4 : apoE4 a une interaction plus forte que apoE3 avec le fragment amyloïdogénique du précurseur de la protéine amyloïde [2] ; en revanche, apoE4 ne se fixe pas à la protéine tau, un composant majeur des faisceaux neuro-fibrillaires, alors que apoE3 y est fortement fixé [3]. La protéine tau stimule l'assemblage et la stabilisation des microtubules; peut-être est-ce par cet intermédiaire que l'apoE3 a un effet positif sur la croissance neuritique. La relation exacte existant entre les effets différentiels d'apoE3 et apoE4 sur les neurones, les microtubules et la protéine amyloïde, et la précocité du déclenchement de la maladie d'Alzheimer, n'est pas encore bien comprise. Cependant, tous ces résultats suggèrent fortement que l'apoE4 est un partenaire direct de la formation des lésions à la base de la maladie d'Alzheimer.

[1. Nathan BP. Science 1994; 264 : 850-2.]

[2. Strittmatter W], et al. Proc Natl Acad Sci USA 1993; 90 : 1977-82.] [3. Strittmatter WJ, et al. Exp Neurol 1994; 125 : 163-72.]

Le gène callipyge du mouton. En 1983, un éleveur de moutons identifia un bélier extrêmement musclé. Cette caractéristique fut transmise à la descendance de ce fondateur, apparemment comme un caractère autosomique dominant, puisque le phénotype hypermusclé se retrouvait chez la moitié des descendants des deux sexes. Comme l'excès musculaire se localise essentiellement dans la région pelvienne, on donna à ce type le nom du callipyge. L'intérêt commercial de cette découverte plus de viande, moins de graisse incita une équipe internationale à entreprendre une étude génétique [1]. Comme peu de marqueurs sont connus chez le mouton, on a utilisé la conservation de séquences et de localisation chromosomique entre ovins et bovins. On s'est donc servi d'une batterie de marqueurs bovins, et on a pu assigner le gène callipyge CLPG au chromosome 18 du mouton (correspondant au 21 bovin). C'est la première étape d'une tentative de clonage positionnel du gène.

[1. Cockett NE, et al. Proc Natl Acad Sci USA 1994; 91 : 3019-23.] 